\title{
Extension of a model for the distribution of voidage around bubbles in a fluidized bed
}

\author{
C. Rautenbach, C. Jayarathna, M. C. Melaaen \& B. M. Halvorsen \\ Institute for Process, Energy and Environmental Technology, \\ Telemark University College, Norway
}

\begin{abstract}
Up to date most models predicting the voidage around bubbles in fluidized beds assume a simple two-phase flow model. A classic example of a model using this basic assumption is the Davidson and Harrison bubble model. In more recent experimental studies it has been shown that there exists a shell of lower voidage around a bubble in a fluidized bed. Better understanding of these phenomena might play a significant role in understanding other very important effects associated with bubbles in fluidized beds. An example of this might be better understanding the wake of a bubble rising in a fluidized bed and also to better predict mixing in a fluidized bed. The amount of contact between the gas and the solids in a gas-solid fluidized bed is paramount for designing an effective fluidized bed reactor.

In the present study a model describing the voidage distribution in front and behind a bubble in a fluidized bed was reexamined and extended. The model proposed by Buyevich et al. used some basic assumptions and previously derived models to compose the model. In the present study the model is extended to two dimensions to give a non-uniform particle distribution around the bubble. Thus voidage distributions all around the bubble could be determined. This new distribution of the voidage could be used to determine a new form of the bubble surface and thus a new boundary condition for the fluidized bubble.

The results from the model are compared with simulations carried out at the TUC in Porsgrunn, Norway. The results are discussed and evaluated. Keywords: solid fraction, voidage distribution, bubble model, fluidization.
\end{abstract}




\section{Introduction}

\subsection{The momentum conservation equation and some basic assumptions}

In some special cases the dispersed phase in a gas-solid fluidized bed may be modeled as a dense gas [3]. The model extended in the present study is based on the closure of the conservation equation for the dispersed phase in a fluidized bed based on this dense gas assumption. Conservation equations for the mass, momentum and fluctuation energy can be postulated in this manner but for the present study only the momentum balance is of interest. The conservation of momentum in the dispersed phase in a fluidized bed can thus be expressed as

$$
\rho_{1}(\phi \partial / \partial t+\langle\phi \mathbf{w}\rangle \cdot \nabla) \mathbf{w}=\nabla \mathbf{P}+n\langle\mathbf{f}\rangle+\phi \rho_{1} \mathbf{g},
$$

with $\rho_{1}$ the particle phase density, $\phi$ the solid fraction, $\mathbf{w}$ the particle velocity, $\mathbf{P}$ the tensor of the particulate stresses and $\mathbf{f}$ the interphase interaction force per particle [3]. The number concentration of particles are represented by $n$ and $\mathbf{g}$ is gravitational acceleration. The averaged values in equation (1), namely the total volume flux, $\langle\phi \mathbf{w}\rangle$, and the random forces experienced by the particles, $\langle\mathbf{f}\rangle$, can be approximated by $\phi \mathbf{w}$ and $\mathbf{f}$ respectfully, given that the fluctuations of these quantities are relatively small [3]. For simplicity of the model these random forces and the total volume flux was assumed as non-fluctuating quantities. Another simplification proposed by Buyevich [3] is to neglect the quasi-viscous stresses. As a result the gradient of particle stresses can be written as $-\nabla p_{1}$, where $p_{1}$ represents the pressure in the dispersed phase. This approximation resembles the ideal-fluid approximation given by Euler [3]. The total conservation of momentum can thus be expressed as

$$
\rho_{1}(\phi \partial / \partial t+\phi \mathbf{w} \cdot \nabla) \mathbf{w}=-\nabla p_{1}+n \mathbf{f}+\phi \rho_{1} \mathbf{g} .
$$

The random forces experienced by the particles consists of three entities namely the drag force, buoyancy and inertial effects which may originate from several different origins.

The drag force per particle can generally be expressed as

$$
\mathbf{f}_{d}=m\left[F_{1}(\phi)+F_{2}(\phi) u\right] \mathbf{u},
$$

with $\mathbf{u}=\mathbf{v}-\mathbf{w}$ and where $\mathbf{v}$ is the interstitial velocity. Thus $\mathbf{u}$ is the fluid slip velocity [3]. In equation (3) $m$ represents the particle mass. Several correlations exist for the prediction of $F_{1}$ and $F_{2}$ as a function of the solid fraction, $\phi$, and other physical parameters. Any adequate drag model can be used and in the present study the model suggested in the work by Buyevich et al. [2] will be used. The functional value of $F_{1}$ will be more significant in the Darcy regime while $F_{2}$ will be more dominant in the Forchheimer regime. In the transition from one regime to the other both terms in the drag correlation will be of importance. For the sake of simplicity low particle Reynolds number flow was assumed in the present model. Thus only the Darcy regime will be of importance and thus $F_{2}$ will be assumed negligible. 
According to Buyevich this correlation for $F_{1}$ can be expressed in terms of a self-consistent model for finally dispersed concentrated suspensions and is given by

$$
F_{1}=\frac{9}{2} \frac{\rho_{0} \mu}{\rho_{1} d_{p}^{2}} \frac{1}{(1-\phi)^{n}},
$$

where $\mu$ is the fluid viscosity, $d_{p}$ is the effective particle diameter and $\rho_{0}$ is the gas density. For this application a value of $\frac{5}{2}$ is assumed for the value of $n$ in equation (4) which corresponds to a value of 4.5 for the exponent in the Richardson and Zaki equation [2].

The contribution of buoyancy to the total force experienced by a particle can be expressed as

$$
\mathbf{f}_{b}=-\frac{4 \pi}{3} d_{p}^{3} \rho(\phi) \mathbf{g}
$$

where $\rho(\phi)=(1-\phi) \rho_{0}+\phi \rho_{1}$ and is representative of the mean density of the mixture.

The last force to be considered is fluid inertia. This force will be ignored in the total interphase interaction force and is an acceptable assumption when working with gas as the ambient fluid [3]. Moreover this force will be much less significant compared to the influence of the drag and buoyancy forces.

Thus the total random interphase interaction force per unit volume of the mixture without fluctuations can be expressed as

$$
n \mathbf{f}=n\left(\mathbf{f}_{d}+\mathbf{f}_{b}\right)=\phi \rho_{1}\left[F_{1}(\phi)+F_{2}(\phi) u\right] \mathbf{u}-\phi \rho(\phi) \mathbf{g}
$$

with $n$ representing the number of identical spherical particles per unit volume of the mixture [3].

\subsection{First approximations and closure}

Following the procedure outlined in the work by Buyevich et al. [2] the Davidson and Harrison model [1] will be used as a first approximation for the particle velocity, w, as well as for the relative interstitial gas velocity, u. According to a simple filtration model for a homogeneous porous body containing a spherical void in two dimensions it follows that

$$
\mathbf{u}=u_{0}\left(1+\frac{2 R^{3}}{r^{3}}\right) \cos \theta \vec{r}-u_{0}\left(1+\frac{R^{3}}{r^{3}}\right) \sin \theta \vec{\theta},
$$

where $R$ is the radius of a sphere with the same volume as the bubble at a particular height in the fluidized bed. Equation (7) is written in spherical coordinates where the radial and angular direction is defined in Figure 1. In the unperturbed dense phase the interstitial velocity will be given by

$$
u_{0}=\left(1-\phi_{0}\right)^{n+1} u_{t}
$$




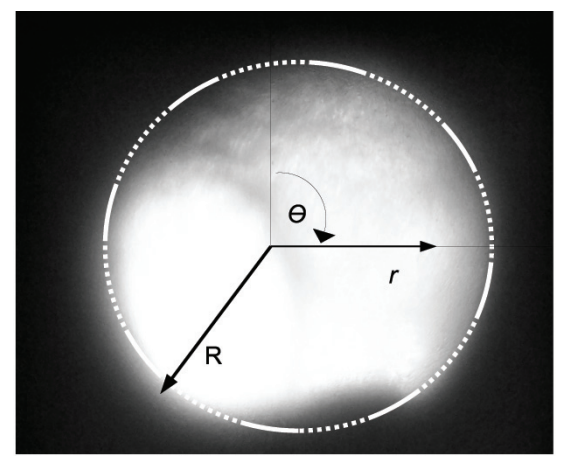

Figure 1: Schematic illustration of the proposed model alongside an image of a real fluidized bubble.

where $u_{t}$ is the terminal fall velocity of a single particle in a stagnant gas and can be expressed as

$$
\frac{\rho_{1}}{\rho_{0}} \frac{2 d_{p}^{2}}{9 \mu} g .
$$

This particular model will be assumed to be adequate in the present study but other correlations might produce better results. Further work on this particular topic is planed in future work. The particle velocity, w, is given by the ideal flow model around a sphere and is expressed as

$$
\mathbf{w}=U_{b}\left\{\left(\frac{R^{3}}{r^{3}}-1\right) \cos \theta \vec{r}+\left(\sin \theta+\frac{R^{3}}{2 r^{3}} \sin \theta\right) \vec{\theta}\right\},
$$

in two dimensions with $U_{b}=2 / 3 \sqrt{g R}$ from the Davidson and Harrison model [1].

The only term in equation (2) that still needs closure is the particulate pressure term. Different approximations also exist for the particulate pressure. In work done by Buyevich [4] models are suggested for the closure of the particular pressure. In the present study the particulate pressure will be assumed to be given by

$$
p_{1}=G(\phi)(\phi / v) T
$$

as described by Buyevich [3]. In equation (11) $\phi / v$ represents the number concentration of particles with $v$ the volume of a sphere. The function $G(\phi)$ describes the increase of the pressure in a dense gas consisting of hard spheres as compared to that of a dilute gas [2]. This function is based on a statistical mechanical model of Carnahan and Starlling [2]. Thus it follows that

$$
G(\phi)=\frac{1+\phi+\phi^{2}-\phi^{3}}{(1-\phi)^{3}} .
$$

In equation (11) the "temperature" of the pseudo-gas is represented by $T$. Again different models may be used to model this analogy to a molecular system and in 
the present study it will be assumed to be given by

$$
(\phi / v) T=6.83 \times 10^{-3} \phi M^{2}\left\langle\phi^{2}\right\rangle \rho_{1} u^{2},
$$

with

$$
M=\frac{n+1}{1-\phi}+\frac{g}{F_{1}(\phi) u} ;\left\langle\phi^{2}\right\rangle=\phi^{2}\left(1+2 \phi \frac{4-\phi}{(1-\phi)^{4}}\right)^{-1} .
$$

In this model for the particular pressure, pulsation energy transfer and work done in expanding the particle pseudo-gas is ignored. Gradients in time and space of average variables are also ignored [2]. From literature it is clear that this particular model is not the best model for predicting the particulate pressure but it will suffice for now as a good starting point for expanding the existing model described by Buyevich et al. [2].

\section{The Buyevich model}

In the original work done by Buyevich et al. [2] only the voidage distribution in one dimension was described. Thus only the voidage directly in front of and at the back of the bubble could be predicted. The present study aimed to use the same basic approach to create a more general model that would be capable of predicting the voidage all around the bubble, in other words, for all values of $r$ and $\theta$ (refer to Figure 1). As a starting point of the extended model only two dimensions will be discussed here. The two dimensional solution was found by solving equation (2) along several different radial lines from the center of the bubble. As an initial control the results were tested with the results published by Buyevich et al. for the voidage along the vertical axis of the bubble [2]. This will be the case with $\theta=0$. In Figure 2 these results are shown for the voidage distribution in front of and at

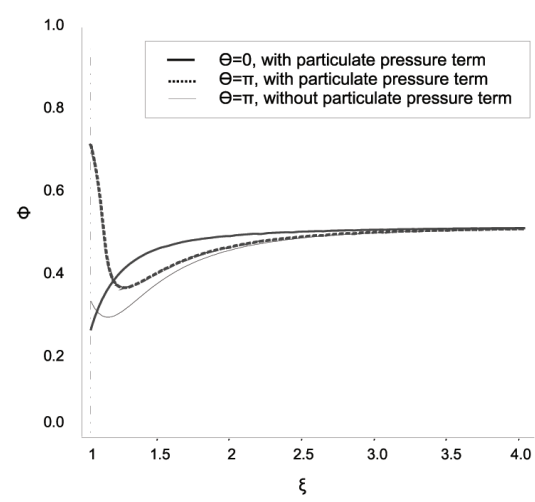

Figure 2: The prediction of the Buyevich bubble model with a free bed solid fraction, $\phi_{0}$, of 0.5 and a bubble radius, $R$, equal to one. The prediction is given in front of- and at the back of the bubble. 
the back of the bubble. In Figure 2 the $\xi$ represents the dimensionless distance and is defined as $\xi=r / R$, with $R$ as the bubble radius.

The radius of the bubble will be taken as a sphere with approximately the same volume as that of the real bubble. In practice it is not always trivial to determine the radius of this equivalent sphere but the concept will be used in the present study. Figure 2 is just a control to check that the new two dimensional equations simplify back to the results obtained by Buyevich et al. [2]. Exactly the same result is retrieved with a solid fraction equal to 0.5 and a bubble radius equal to one. Some differences were observed in the prediction at the rear of the bubble. The results published by Buyevich did not include the particulate pressure term in the total conservation of momentum equation. In some cases it is acceptable to assume that this term is negligible but it is clear that this term has a significant effect on the prediction of the model. In front of the bubble the results of the model seems fairly physical and is a monotonously increasing function of the solid fraction. The only effect of neglecting the particulate pressure term here would be a higher solid fraction at the bubble boundary. At the back of the bubble the particulate terms causes a very high solid fraction at the bubble interface. Whether this is physical or not is not clear as a solid fraction of 0.7 seems very high if the free bed solid fraction is a mere 0.5 . Further research on the physicality of this phenomena is needed.

\section{Simulation correlations with the two dimensional model}

In an attempt to quantify the results obtained with the model around the bubble, CFD simulations were used to produce data. The simulation data should give some indication of the validity of the model in question. The commercial code Fluent Ansys 12.1 was used to perform the simulations. The Eularian approach was employed and the Syamlal et al. drag model was used to simulate the drag [5]. A uniform particle size equal to $483 \mu \mathrm{m}$ was assumed and the material was chosen as glass. This set-up created a fluidized bed with a free bed solid fraction, $\phi_{0}$, of 0.63 . The fluidization medium was modeled as air. All of the simulations carried out was in a two dimensional rectangular column with a diameter of $23 \mathrm{~cm}$.

In Figure 3(a) a bubble is shown that was retrieved using simulations. To produce a single bubble the bed was modeled to be at minimum fluidization conditions and then an extra supply of gas was fed into the bed via a jet that was located in the middle of the bed.

The data was extracted as several points along several radial lines drawn from the estimated center of the bubble. The radial lines that were investigated are given in Figure 3(b) and the particular point used are illustrated in Figure 3(c). At each point the solid fraction was extracted. The data from the extracted points were compared with the model along each radial line in term of the dimensionless distance, $\xi$. The results are given in Figure 4. The radial lines that were investigated are located at $\theta=0, \pi / 6, \pi / 3$ and $\pi / 2$ respectively (refer to Figure 3(b)). The equivalent spherical bubble radius was taken as $7 \mathrm{~cm}$. This is an empirical estimation. A definition for the bubble boundary should also be defined. 


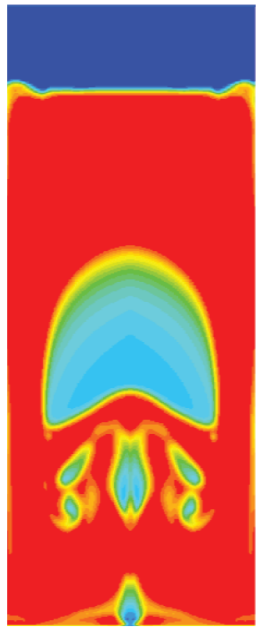

(a)

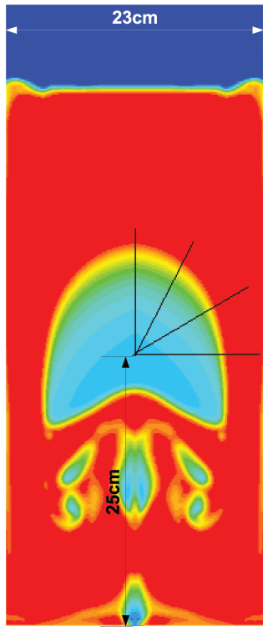

(b)

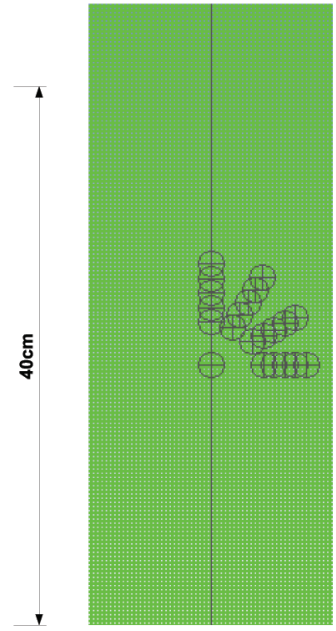

(c)

Figure 3: (a) A bubble created in a fluidized bed at minimum fluidization conditions, (b) radial lines investigated to quantify the accuracy of the model and (c) the point at which the void fraction data were extracted.

From Figure 4(a)-(b) it is clear to see that the correlation without the particulate pressure term provides a much more accurate result. This may be a misleading result as the value of $\xi$ is strongly connected to what is defined as the bubble boundary. If the equivalent spherical bubble's radius was taken as less that $7 \mathrm{~cm}$ the correlation with the particulate term might have been a better fit. Simply from this result it is clear that further research is needed in clearly defining the bubble boundary.

There were a few unexpected and interesting results from the simulations. First of all the solid fraction inside of the bubble was much higher than expected. In most models commonly used, a simple two phase model is assumed. In other words, there is only a uniform dense phase outside of the bubble and the gas phase inside of the bubble. All gas in excess of the minimum fluidization velocity is assumed to pass through the bed as bubbles [6]. This assumption seems to be very crude in light of the simulations. These phenomena also make it more difficult to define the bubble boundary. Even at what was assumed to be the center point of the bubble a solid fraction of 0.12 was observed. From Figure 4 it is clear to see that the first two data points on each radial line had a some what different gradient than the other data points. This change in gradient might be an indication of the bubble boundary, in which case, the new two dimensional model does relatively well in prediction the boundary. Contrary to the previous published results it seems that the bubble boundary cannot always be assumed to start at a solid fraction of zero [2]. As the angle, $\theta$, was tending toward $\pi / 2$ the model correlation became worse (refer to Figure 4(c) and (d)). Actually the model predictions depicted in Figure 


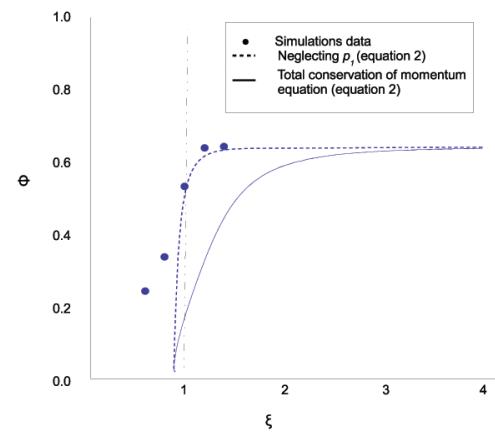

(a)

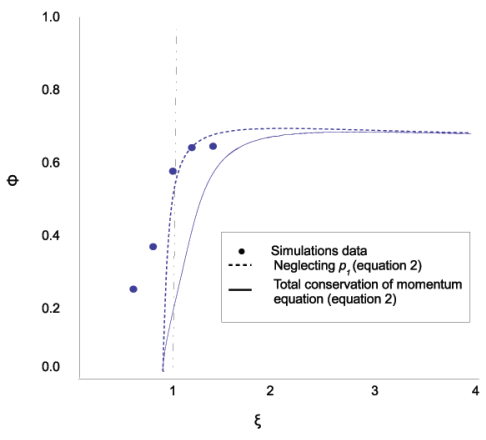

(c)

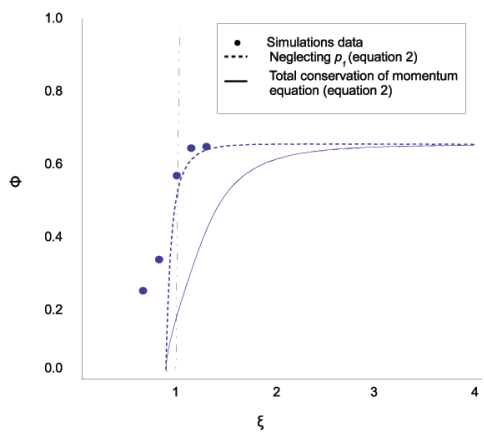

(b)

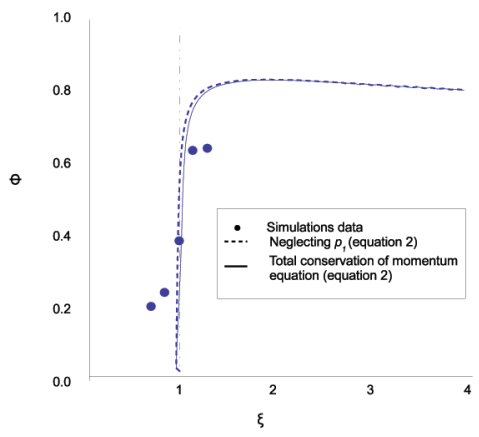

(d)

Figure 4: Solid fraction distribution along the radial lines (a) $\theta=0$, (b) $\theta=\pi / 6$, (c) $\theta=\pi / 3$ and (d) $\theta=\pi / 2$.

4(d) were calculated using the value $\pi / 2.1$ as the model produced a solid fraction distribution equal to zero at $\pi / 2$. This result is unphysical and is a consequence of the trivial model used to predict the relative interstitial velocity, $\mathbf{u}$. From equation (7) it is clear to see that the velocity will be zero at $\pi / 2$ in the radial direction and when the velocity is modeled as zero it follows that the drag and consequently the solid fraction will also be modeled as zero. This problem at $\pi / 2$ raises doubts about the physicality of the model's perditions as $\theta \rightarrow \pi / 2$. This phenomena might also be the explanation for the relatively bad predictions as $\theta \rightarrow \pi / 2$ (refer to Figure 4(c)).

In Figure 5(a) and (b) the same result are show as in Figure 4 but on a bigger scale.

In Figure 5 is a section of higher solid fraction just adjacent to the bubble boundary. Further research on the physicality of this result is in progress. If it can be proven that this region of higher solid fraction does indeed exist, it might help the scientific community understand bubble interaction and coalescence in a more clear way. 


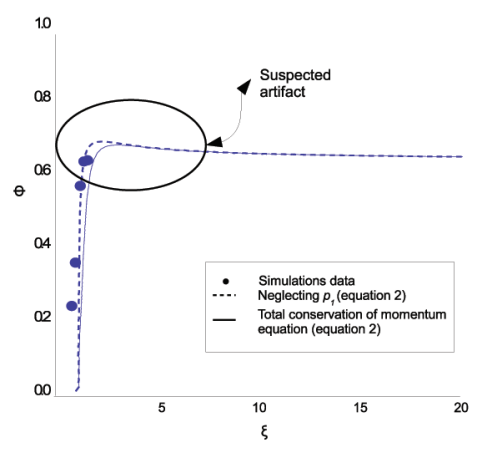

(a)

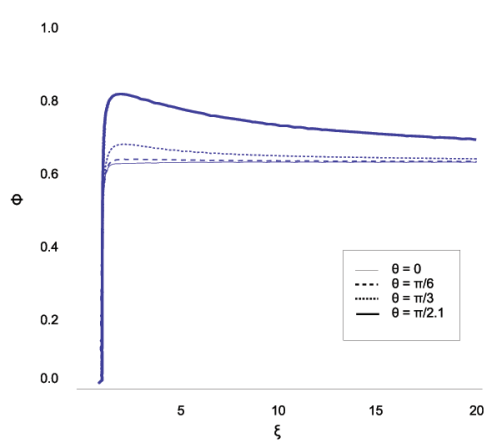

(b)

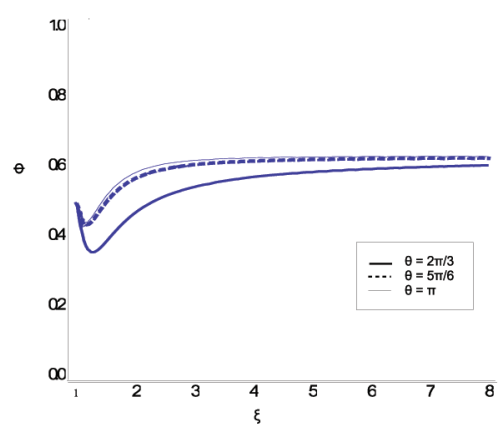

(c)

Figure 5: (a) The solid fraction distribution along the radial line at $\theta=\pi / 3$, (b) the solid fraction distributions along several radial lines and (c) gives the solid fraction distribution in the rear quadrant of the bubble.

In Figure 5(c) the model predictions for $\theta=2 \pi / 3,5 \pi / 6$ and $\pi$ are given. In this quadrant behind the bubble the opposite effect is observed. Adjacent to the hypothetical bubble boundary a band of lower solid fractions exists. This effect is again more pronounced for values of $\theta$ close to $\pi / 2$. Whether this is just an artifact or a real wake effect is not yet clear as no data was acquired in this region.

\section{Conclusion}

Bubbles in fluidized beds are one of the major phenomena through which mixing takes place. Better understanding of physical phenomena in and around bubbles in a fluidized bed is of paramount importance. Knowledge in this regard can help engineers and scientists to design and develop more effective fluidized bed reactors.

In the present study a model describing the voidage distribution in front and behind a bubble in a fluidized bed was reexamined and extended. The model 
suggested is not complete or satisfactory. This work is only the initialization of a research project to model the flow behavior in and around a bubble in a fluidized bed. This model, originally suggested by Buyevich et al. [2], might be developed into a very useful and convenient predictive tool.

Time the model gives very good results in the area close to the front of the bubble. This was established by using data that was acquired using CFD simulations. Suspected limitations of the models used to predict the relative interstitial velocities, equation (7), might of been the cause of the inaccuracy of the model at $\theta=\pi / 2$. Never the less, these problems have the possibility of being addressed in the future and thus render a very accurate and useful model for predicting the solid fraction distribution all around a bubble in a fluidized bed.

\section{References}

[1] Davidson, J.F. \& Harrison, D., Fluidized particles. Cambridge University Press: Cambrige, U.K., 1963.

[2] Buyevich, Y.A., Yates, J.G., Cheesman, D.J. \& Wu, K.T., A model for the distribution of voidage around bubbles in a fluidized bed. Chemical Engineering Science, 50(19), pp. 3155-3162, 1995.

[3] Buyevich, Y.A., Fluid dynamics of coarse dispersions. Chemical Engineering Science, 49(8), pp. 1217-1228, 1994.

[4] Buyevich, Y.A., Particulate pressure in monodisperse fluidized beds. Chemical Engineering Science, 52(1), pp. 123-140, 1997.

[5] Syamlal, M., Rogers, W. \& O'Brien, T.J., Technical note. MFIX Documentation theory guide, U.S. Department of Energy, Office of Fossil Energy, Morgantown Energy Technology Center, Morgantown, West Virginia, 1993.

[6] Kunii, D. \& Levenspiel, O., Fluidization Engineering second edition. Butterworth-Heinemann series in chemical engineering: Oxford, U.K., 1991. 